\title{
Geometric and process design of ultra-thin junctionless double gate vertical MOSFETs
}

\author{
K. E. Kaharudin, F. Salehuddin, A. S. M. Zain, Ameer F. Roslan \\ MiNE, Centre for Telecommunication Research and Innovation, Faculty of Electronics and Computer Engineering, \\ Universiti Teknikal Malaysia Melaka (UTeM), Hang Tuah Jaya, Durian Tunggal, 76100 Melaka, Malaysia
}

\section{Article Info \\ Article history: \\ Received Sep 10, 2018 \\ Revised Mar 15, 2019 \\ Accepted Apr 4, 2019 \\ Keywords: \\ Channel doping \\ Channel length \\ Doping concentration \\ Pillar thickness \\ Source/drain doping \\ Work function}

\begin{abstract}
The junctionless MOSFET architectures appear to be attractive in realizing the Moore's law prediction. In this paper, a comprehensive 2-D simulation on junctionless vertical double-gate MOSFET (JLDGVM) under geometric and process consideration was introduced in order to obtain excellent electrical characteristics. Geometrical designs such as channel length $\left(\mathrm{L}_{\mathrm{ch}}\right)$ and pillar thickness $\left(T_{p}\right)$ were considered and the impact on the electrical performance was analyzed. The influence of doping concentration and metal gate work function (WF) were further investigated for achieving better performance. The results show that the shorter $\mathrm{L}_{\mathrm{ch}}$ can boost the drain current (ID) of n-JLDGVM and p-JLDGVM by approximately $68 \%$ and $70 \%$ respectively. The ID of the n-JLVDGM and p-JLVDGM could possibly boost up to $42 \%$ and $78 \%$ respectively as the $\mathrm{T}_{\mathrm{p}}$ is scaled down from $11 \mathrm{~nm}$ to $8 \mathrm{~nm}$. The channel doping $\left(\mathrm{N}_{\mathrm{ch}}\right)$ is also a critical parameter, affecting the electrical performance of both n-JLDGVM and p-JLDGVM in which 15\% and 39\% improvements are observed in their respective ID as the concentration level is increased from $1 \mathrm{E} 18$ to $9 \mathrm{E} 18$ atom $/ \mathrm{cm}^{3}$. In addition, the adjustment of threshold voltage can be realized by varying the metal WF.
\end{abstract}

Copyright (c) 2019 Institute of Advanced Engineering and Science. All rights reserved.

\section{Corresponding Author:}

F. Salehuddin,

Centre for Telecommunication Research and Innovation,

Faculty of Electronics and Computer Engineering,

Universiti Teknikal Malaysia Melaka (UTeM),

Hang Tuah Jaya, Durian Tunggal, 76100 Melaka, Malaysia.

Email: fauziyah@utem.edu.my

\section{INTRODUCTION}

For more than a decade, Moore's law has been an ultimate guideline for MOSFET minituarization. The main obstacle of shrinking the dimension of MOSFET is the decrease in gate controllability over the channel due to high electric field [1-4]. An alternative solution to improve the gate control over the channel is by adding an extra gate [5-10]. Multi-gate MOSFETs have been recognized promising candidates for future scaling of MOSFET technologies. These multi-gate devices, apart from providing better immunity of short channel effects (SCE), are still not really perfect and suffered from similar challenges encountered with conventional bulk MOSFET. The most crucial challenge is to form an ultra-shallow source/drain (S/D) junctions with high doping concentration gradient, which needs an advanced S/D and channel engineering [11]. Recently, a lot of junctionless MOSFET structures [12-19] have been introduced to overcome the difficulties in forming ultra-shallow junctions. The main design criteria of junctionless MOSFET is that the channel and S/D region are doped with the same dopant type (n- or p-type) and are normally employed in the range of $10^{18}$ to $10^{20} \mathrm{~cm}^{-3}$ [20]. There is no junction formed between the channel and S/D regions, thereby simplifying the fabrication process. Junctionless MOSFETs work in depletion mode, which significantly rely on the ultra-thin body architecture. 
Initial work reported in [21] revealed that the physics of junctionless MOSFET is different from the conventional MOSFET. Current conduction in Junctionless MOSFETs is based on bulk phenomena in which the gate voltage would induce the depleted region in the ultra-thin channel in order to turn off the transistor. Meanwhile, the channel region (ultra-thin body) should be highly doped in order to turn on the transistor instantly. The simulation comparison between Junctionless nanowires transistor (JNT) and the inversion mode multi-gate FET (MuGFET) was reported in [21] and it was conducted using ATLAS module of Silvaco. The results revealed that the subthreshold swing (SS) of the JNT was $21 \%$ lower than MuGFET, implying that the JNT has faster switching capability than MuGFET. This happens due to the utilization of high doping and ultra-thin body in JNT structure that allows a higher electron mobility in the channel. A novel design analysis for a junctionless double gate vertical MOSFET (JLDGVM) using Silvaco TCAD (ATLAS) was previously reported in [22]. The results found that the drain barrier lowering (DIBL), subthreshold swing (SS) and leakage current $\left(\mathrm{I}_{\mathrm{OFF}}\right)$ of the junctionless device are much lower than the junction device. This might happen due to the absence of the junction in which the depeletion layer width at drain and source was totally eliminated. Furthermore, the application of metal-gate and hafnium dioxide $\left(\mathrm{HfO}_{2}\right)$ to the JLDGVM structure did contribute a significant improvement of the on-current $\left(\mathrm{I}_{\mathrm{ON}}\right)$. The metalgate work function (WF) was tuned to obtain a higher $\mathrm{I}_{\mathrm{ON}}$ magnitude for much better performance of the device. Although, the that junctionless vertical configuration has contributed a significant boost on the electrostatic performance, it still requires in-depth investigation on the impact of geometrical and process parameter variation (i.e. channel length, pillar thickness, channel doping, source/drain doping and metal-gate workfunction) on the electrostatic performance of the device. Thus, this recent study will provide a detailed approach of designing, studying, analyzing and improving transistor performance using Silvaco TCAD simulation tools. It will also provide a significant in sight on the design consideration of the JLDGVM in regard with the electrostatic behaviours such as drain current, current density and subthreshold swing.

\section{ULTRA-THIN JLDGVM's DESIGN}

A 2-D simulation for ultra-thin JLDGVMs were investigated, and the process flow of the device is depicted in Figure 1. The device process includes the ultra-thin pillar formation, the high- $k$ metal-gate (HKMG) deposition, source/drain implantation and metallization.

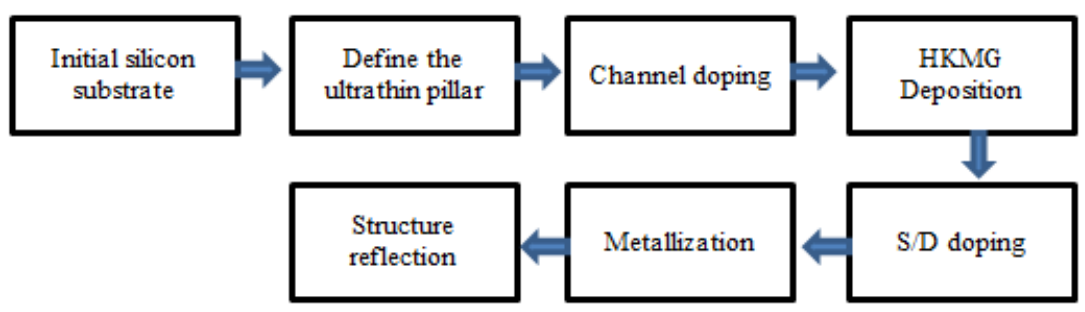

Figure 1. Simulated process flow for ultra-thin JLDGVM's design

\subsection{JLDGVM's structure}

The ultra-thin JLDGVMs utilized hafnium dioxide $\left(\mathrm{HfO}_{2}\right)$ as a dielectric combined with metal-gate, tungsten silicide $\left(\mathrm{WSi}_{\mathrm{x}}\right)$. The simulated $\mathrm{n}$-JLDGVM is a heavily $\mathrm{n}$-type doped $\left(1 \times 10^{18} \mathrm{~cm}^{-3}\right.$ of Arsenic) channel with $10-7 \mathrm{~nm}$ range of silicon pillar's thickness $\left(\mathrm{T}_{\mathrm{p}}\right)$ and $15-12 \mathrm{~nm}$ range of channel length $\left(\mathrm{L}_{\mathrm{ch}}\right)$. In contrast, the simulated p-JLDGVM is heavily doped with $1 \times 10^{18} \mathrm{~cm}^{-3}$ of boron dose. Double-gate structure was used to provide better controllability over the channel. The source and drain region for both $n$ and $p$-type device were also heavily doped with the similar type as channel doping in order to form $\mathrm{N}^{+} \mathrm{N}^{+} \mathrm{N}^{+}$and $\mathrm{P}^{+} \mathrm{P}^{+} \mathrm{P}$. Table 1 shows the geometrical and process parameter values used in the simulated $n$-channel and $\mathrm{p}$-channel devices. The simulation was conducted by varying one parameter at a time while keeping the other parameters constant in order to study the effect of a specific design parameter on the device performance. The 2-D crosssection of the device which define the physical parameters like silicon pillar's thickness $\left(\mathrm{T}_{\mathrm{p}}\right)$ and channel length $\left(\mathrm{L}_{\mathrm{ch}}\right)$ is depicted in Figure 2. 
Table 1. Parameters used in the simulated JLDGVMs

\begin{tabular}{cccc}
\hline Variables & Units & Fixed value & Manipulated range \\
\hline Channel Length, $\mathrm{L}_{\mathrm{ch}}$ & $\mathrm{nm}$ & 11 & 9 to 12 \\
Pillar Thickness, $\mathrm{L}_{\mathrm{p}}$ & $\mathrm{nm}$ & 9 & 11 to 8 \\
Channel doping, $\mathrm{N}_{\mathrm{ch}}$ & Atom $/ \mathrm{cm}^{3}$ & $1.0 \mathrm{E} 18$ & $1.0 \mathrm{E} 18$ to $9.0 \mathrm{E} 18$ \\
S/D doping, $\mathrm{N}_{\mathrm{sd}}$ & Atom $/ \mathrm{cm}^{3}$ & $1.0 \mathrm{E} 18$ & $1.0 \mathrm{E} 18$ to $9.0 \mathrm{E} 18$ \\
Metal work-function, WF & $\mathrm{eV}$ & 4.5 & 4.5 to 4.7 \\
\hline
\end{tabular}

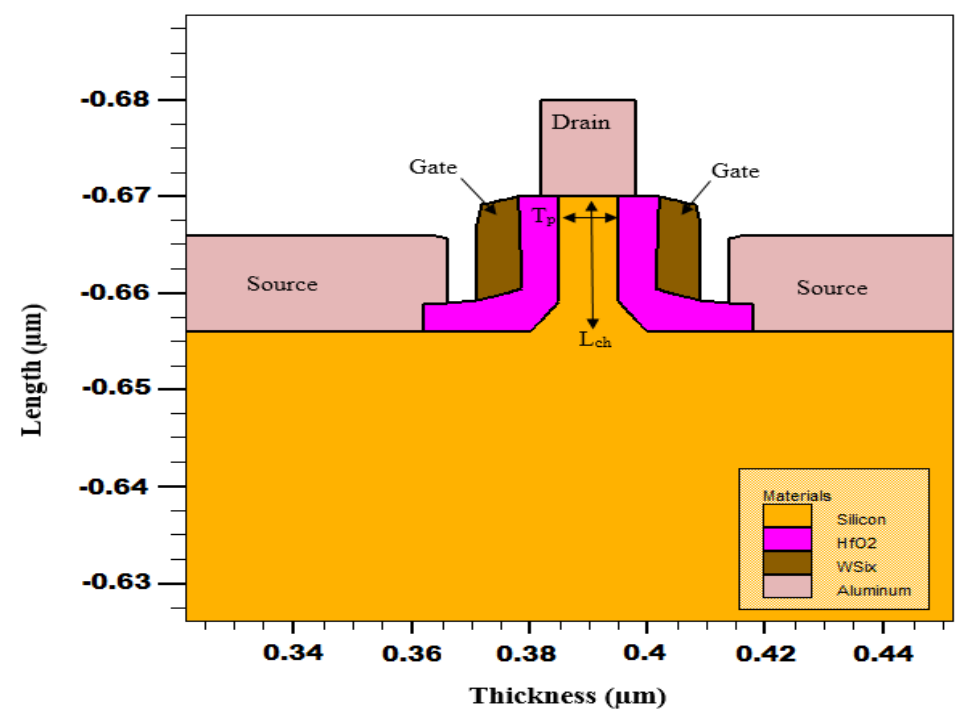

Figure 2. Cross section of the JLDGVM device

\subsection{Device simulation}

The TCAD Silvaco, Athena and Atlas were employed in this work to simulate the electrical characteristics of the JLDGVM devices. The devices were set up for simulation in both linear and saturation mode. For the purpose of simulating I-V curves of the devices, the Silvaco TCAD utilizes the following set of physical model: 1) drift diffusion (DD) model with simplified Boltzmann carrier statistics; 2). The inversion layer mobility model combined with SRH (Shockley-Read-Hall Recombination) with fixed carrier lifetimes models. This recombination model was selected to consider the phonon transitions effect due to the presence of a trap (or defect) within the forbidden gap of the devices. Critical electrical characteristics like threshold voltage $\left(\mathrm{V}_{\mathrm{TH}}\right)$ ON-state current $\left(\mathrm{I}_{\mathrm{ON}}\right)$, OFF-state current $\left(\mathrm{I}_{\mathrm{OFF}}\right)$ and subthreshold swing (SS) could be extracted from the $I_{D}$ vs. $V_{G}$ characteristic. The device simulation condition is set up as listed in Table 2 [23]. The $\mathrm{I}_{\mathrm{ON}}$, I $\mathrm{OFF}$ and SS values can be extracted from the curve.

Table 2. Device simulation conditions [23]

\begin{tabular}{ccccc}
\hline \multirow{2}{*}{ Electrical Characteristics } & \multirow{2}{*}{ Drain Voltage, $\mathrm{V}_{\mathrm{D}}(\mathrm{V})$} & \multicolumn{3}{c}{ Gate Voltage, $\mathrm{V}_{\mathrm{G}}(\mathrm{V})$} \\
& & $\mathrm{V}_{\text {Initial }}$ & $\mathrm{V}_{\text {Step }}$ & $\mathrm{V}_{\text {Final }}$ \\
\hline Threshold Voltage $\left(\mathrm{V}_{\mathrm{TH}}\right)$ & 1.0 & 0 & 0.1 & 2.0 \\
On-state Current $\left(\mathrm{I}_{\mathrm{ON}}\right)$ & 1.0 & 0 & 0.1 & 2.0 \\
Off-state Current (I $\left.\mathrm{I}_{\mathrm{OFF}}\right)$ & 1.0 & 0 & 0.1 & 2.0 \\
Subthreshold Swing (SS) & 1.0 & 0 & 0.1 & 2.0 \\
\hline
\end{tabular}

\section{GEOMETRIC DESIGN CONSIDERATION}

Figure 3 depicts the impact of $L_{c h}$ and $T_{p}$ on $I_{D}$ vs. $V_{G}$ characteristics of the JLDGVM devices. Figure 3(a) shows the impact of channel length $\left(\mathrm{L}_{\mathrm{ch}}\right)$ scaling on the $\mathrm{I}_{\mathrm{D}}-\mathrm{V}_{\mathrm{G}}$ characteristic of both n-JLVDGM and p-JLVDGM devices. The drain current $\left(\mathrm{I}_{\mathrm{D}}\right)$ of the n-JLVDGM and p-JLVDGM can boost up to $68 \%$ and $70 \%$ respectively as the $L_{c h}$ is scaled down from $12 \mathrm{~nm}$ to $9 \mathrm{~nm}$, shifting the $\mathrm{I}_{\mathrm{D}}-\mathrm{V}_{\mathrm{G}}$ characteristic to the positive $\mathrm{x}$-axis for $\mathrm{n}$-JLVDGM and to the negative $\mathrm{x}$-axis for $\mathrm{p}$-JLVDGM. In this work, the smaller $\mathrm{L}_{\mathrm{ch}}$ enhances the current flow due to less resistance resulted from shorter path between source and drain region. As a result, the $V_{T H}$ decreases, allowing the $I_{D}$ to significantly increase. Both $n$ - and p-type devices show that the variation of $L_{c h}$ do contribute a significant impact on the $I_{D}$ as the maximum $I_{D}$ is increased over

Geometric and process design of ultra-thin junctionless double gate vertical MOSFETs (K. E. Kaharudin) 
the reduction of $\mathrm{L}_{\mathrm{ch}}$. Meanwhile, the drain current $\left(\mathrm{I}_{\mathrm{D}}\right)$ of the n-JLVDGM and p-JLVDGM could boost up to $42 \%$ and $78 \%$ respectively as the $T_{p}$ is scaled down from $11 \mathrm{~nm}$ to $8 \mathrm{~nm}$, shifting the $\mathrm{I}_{D^{-}}-V_{G}$ characteristic to the positive $\mathrm{x}$-axis for $\mathrm{n}$-JLVDGM and to the negative $\mathrm{x}$-axis for p-JLVDGM. The thiner pillar would speed up the device as the channel is rapidly transformed to fully-depleted mode.

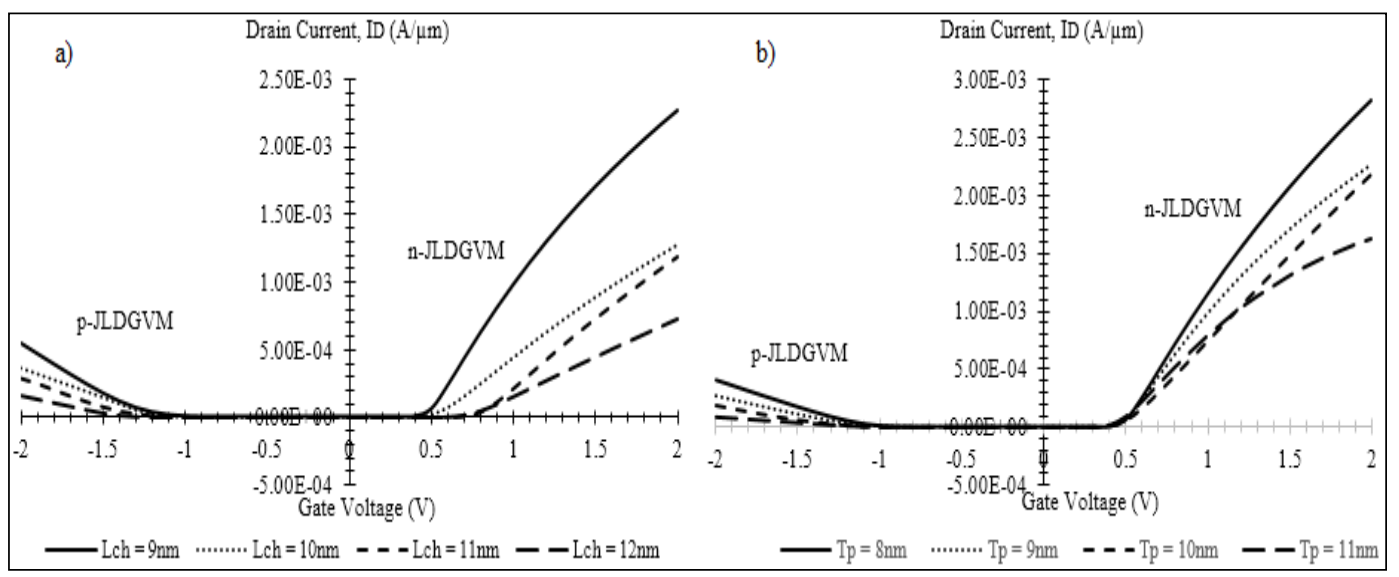

Figure 3. Graph of the drain current $\left(\mathrm{I}_{\mathrm{D}}\right)$-gate voltage $\left(\mathrm{V}_{\mathrm{G}}\right)$ at $\mathrm{V}_{\mathrm{D}}=1.0 \mathrm{~V}$ with different $(\mathrm{a}) \mathrm{L}_{\mathrm{ch}}$ and $\left.\mathrm{b}\right) \mathrm{T}_{\mathrm{p}}$

The subthreshold swing (SS) is an important characteristic of MOSFETs that measure how fast the transition could be triggered from 'ON' to 'OFF' condition or vice versa. It also determines the scalability limits of the devices by indicating how effectively the flow of drain current could be halted as the gate voltage is decreased below threshold limit [24]. The subthreshold swing (SS) was extracted from the inverse slope of $\log _{10} I_{D}$ vs $V_{G S}$ characteristic shown in (1) [24]:

$$
S S=\left[\frac{d\left(\log _{10} I_{D S}\right)}{d V_{G S}}\right]^{-1}
$$

Figure 4 depicts the impact of the channel length variation on the SS characteristic of the devices. The SS is slightly decreased as the channel length of the devices are deliberately decreased, implying a directly proportional relationship between $\mathrm{SS}$ and $\mathrm{L}_{\mathrm{c}}$ for both $\mathrm{n}$ - and $\mathrm{p}$-channel JLDGVM. The shorter $\mathrm{L}_{\mathrm{c}}$ of the device emulates the behavior of a fully-depleted devices in which the majority carrier (electron for n-JLDGVM and holes for p-JLDGVM) concentration were depleted as a sufficient gate voltage is applied. This subsequently reduces the parasitic capacitance which offers a near ideal SS characteristic (60mV/decade).

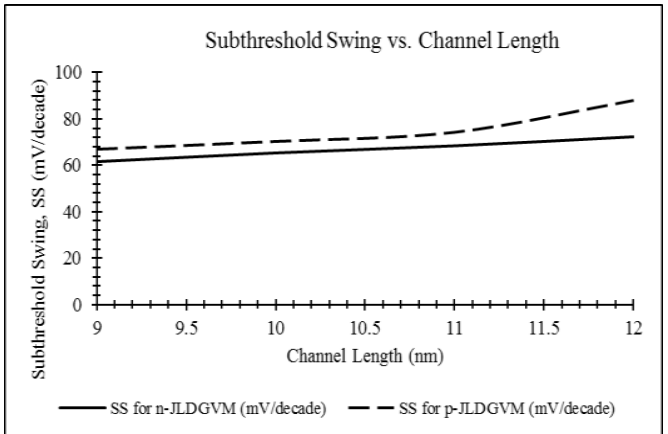

Figure 4. Subthreshold swing vs. channel length

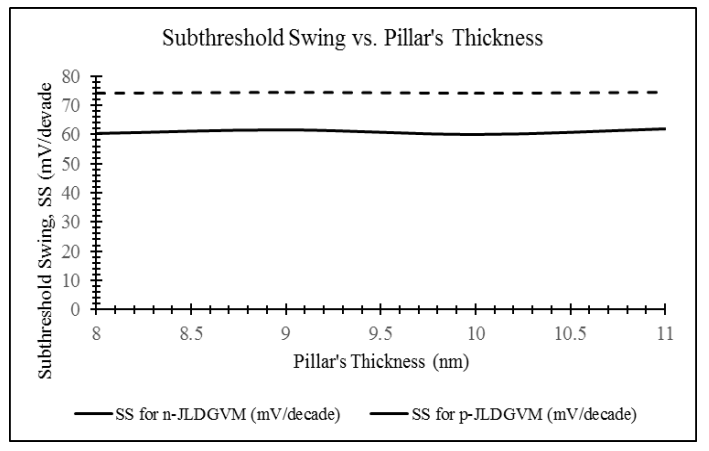

Figure 5. Subthreshold swing vs. pillar thickness 
Figure 5 depicts the impact of the pillar thickness variation on the SS characteristic of the devices. It is observed that the variation in the pillar thickness variation do not contribute much effect on the SS characteristic. This is mainly due to the presence of high- $k /$ metal-gate stack in forming the conducting channel of the device. The utilization of tungsten silicide $\left(\mathrm{WSi}_{\mathrm{x}}\right)$ and hafnium dioxide $\left(\mathrm{HfO}_{2}\right)$ as the substitute materials for polysilicon and $\mathrm{SiO}_{2}$ accordingly do reduce the amount of the required gate voltage to increase the drain current per decade. Since there are no poly depletion effects in high- $k / \mathrm{metal}$-gate configuration, the variation in the pillar thickness is almost insignificant.

\section{PROCESS DESIGN CONSIDERATION}

For further understanding of the behavior of JLDGVMs, the impact of process design variation on $\mathrm{I}_{\mathrm{D}}-\mathrm{V}_{\mathrm{G}}$ is presented in this section. Section $\mathrm{A}, \mathrm{B}$, and $\mathrm{C}$ will describe the impact of channel doping $\left(\mathrm{N}_{\mathrm{ch}}\right)$, source/drain doping and work function (WF) upon the drain current $\left(\mathrm{I}_{\mathrm{D}}\right)$, leakage current $\left(\mathrm{I}_{\mathrm{OFF}}\right)$ and subthreshold swing respectively.

\subsection{Channel doping $\left(\mathrm{N}_{\mathrm{ch}}\right)$}

Channel doping is a common approach to tune the $\mathrm{V}_{\mathrm{TH}}$ of MOSFET devices [16],[25]. For short channel devices, the doping concentration in the channel region play a crucial part in determining the maximum $I_{D}$, $I_{O F F}$ and also the $S S$ characteristics. For that reason, multiple $I_{D}-V_{G}$ curves with respect to different $\mathrm{N}_{\mathrm{ch}}$ concentration are investigated, as depicted in Figure 6. Based on the curves, it can be observed that the higher concentration of $\mathrm{N}_{\mathrm{ch}}$ reduces the $\mathrm{V}_{\mathrm{TH}}$ value in both $\mathrm{n}$-channel and p-channel JLDGVM devices. This is mainly due to mobility enhancement resulted from the increase of majority carriers in the channel region. As a results, the maximum $\mathrm{I}_{\mathrm{D}}$ is observed to be improved by approximately $15 \%$ and $39 \%$ in both n-channel and p-channel JLDGVM devices respectively as a higher concentration of $\mathrm{N}_{\mathrm{ch}}$ is applied. In other word, the higher concentration of $\mathrm{N}_{\mathrm{ch}}$ allows much lower gate voltage to invert the channel. Nevertheless, such low $\mathrm{V}_{\mathrm{TH}}$ causes deterioration in leakage current ( $\mathrm{I}_{\mathrm{OFF}}$ ) as depicted in Figure 7. From the graph, it can be observed that the higher concentration of $\mathrm{N}_{\mathrm{ch}}$ do increase the IofF value of both devices. Such occurrence mainly happens due to higher subthreshold slope that slow down the variation of $I_{D}$ that occurs below the $\mathrm{V}_{\mathrm{TH}}$. Figure 8 depicts the impact of channel doping concentration upon SS variation.

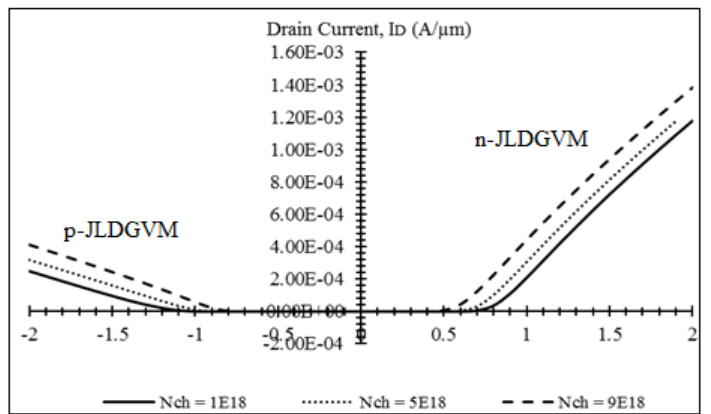

Figure 6. Graph of the drain current $\left(\mathrm{I}_{\mathrm{D}}\right)$-gate voltage $\left(\mathrm{V}_{\mathrm{G}}\right)$ at $\mathrm{V}_{\mathrm{D}}=1.0 \mathrm{~V}$ with different concentration of channel doping

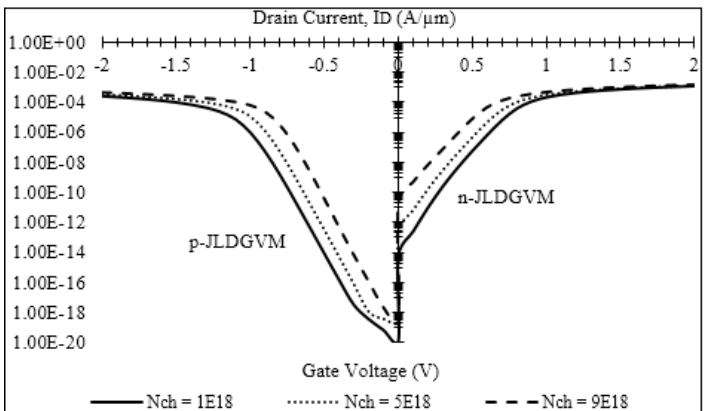

Figure 7. Graph of the subthreshold drain current $\left(\mathrm{I}_{\mathrm{D}}\right)$-gate voltage $\left(\mathrm{V}_{\mathrm{G}}\right)$ at $\mathrm{V}_{\mathrm{D}}=1.0 \mathrm{~V}$ with different concentration of channel doping

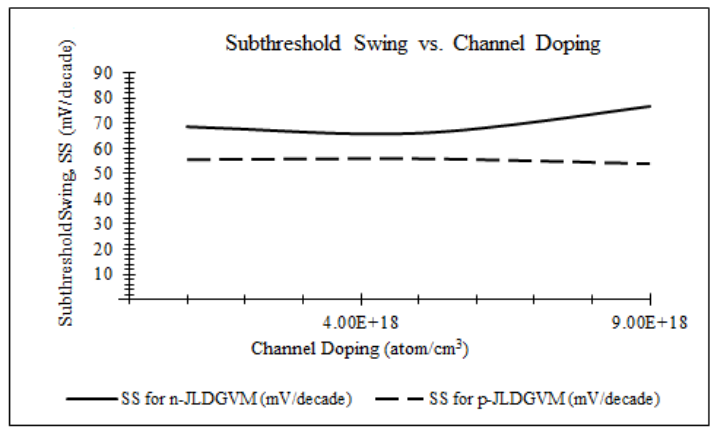

Figure 8. Subthreshold swing vs. channel doping 
Based on the plot, the SS variation of n-JLDGVM is more prone to roll up along with an increased $\mathrm{N}_{\mathrm{ch}}$ compared to p-JLDGVM. This is fundamentally due to the electrons in n-channel have twice mobility acceleration compared to holes in p-channel. The SS of n-JLDGVM is observed to be approximately $19 \%$ higher than the SS of p-JLDGVM. This indicates that the p-JLDGVM could switch from its off-state to onstate much faster than the n-JLDGVM.

\subsection{Source/drain (S/D) doping (Nsd)}

In junctionless configuration, the material used for the source/drain doping is similar with the material used in previous channel doping process [11]. For instance, the n-JLDGVM and p-JLDGVM fabrication requires arsenic and boron respectively as dopants for source/drain doping $\left(\mathrm{N}_{\mathrm{sd}}\right)$. Figure 9 presents the variation effects of $\mathrm{N}_{s d}$ on the $\mathrm{I}_{\mathrm{D}}-\mathrm{V}_{\mathrm{G}}$ characteristic for both JLDGVM and p-JLDGVM devices. In short channel devices, the diffusion of the $\mathrm{S} / \mathrm{D}$ doping play an important role in $\mathrm{V}_{\mathrm{TH}}$ changes. However, the variation of $N_{s d}$ in JLDGVM devices do not give any significant impact on the $I_{D^{-}}-V_{G}$ characteristic. This obviously shows that the concentration of $\mathrm{N}_{\mathrm{sd}}$ in both devices is too low to change the $\mathrm{V}_{\mathrm{TH}}$. The values of $\mathrm{N}_{\mathrm{sd}}$ are chosen below $10^{20} \mathrm{~cm}^{-3}$ in order to limit band-to-band-tunneling (BTBT) current due to heavy $\mathrm{N}_{\mathrm{ch}}$ of $10^{18} \mathrm{~cm}^{-3}$. The higher doping concentration in the silicon channel triggers larger effect of BTBT which eventually leads to a significant leakage current in the OFF state [3]. As a results, the maximum $\mathrm{I}_{D}$ is enhanced by approximately $10.6 \%$ and $2.3 \%$ in both n-channel and p-channel JLDGVM devices respectively as a higher concentration of $\mathrm{N}_{\mathrm{sd}}$ is applied.

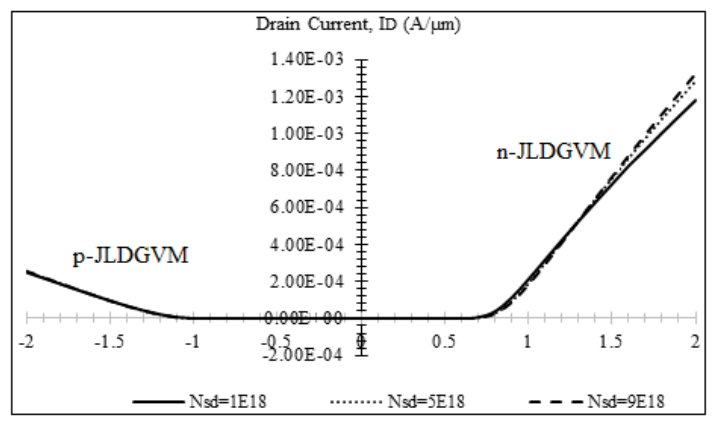

Figure 9. Graph of the drain current $\left(\mathrm{I}_{\mathrm{D}}\right)$-gate voltage $\left(\mathrm{V}_{\mathrm{G}}\right)$ at $\mathrm{V}_{\mathrm{D}}=1.0 \mathrm{~V}$ with different concentration of S/D doping

Further observation shows that further leakage occurs when $\mathrm{N}_{\mathrm{sd}}$ is increased due to $\mathrm{V}_{\mathrm{TH}}$ reduction, as depicted in Figure 10. The reduction in leakage current $\left(\mathrm{I}_{\mathrm{OFF}}\right)$ for $\mathrm{p}-\mathrm{JLDGVM}$ as the $\mathrm{N}_{\mathrm{sd}}$ is increased is extremely minimal. The changes in IOFF values for p-JLDGVM is almost unnoticable where the percentage difference is only $28 \%$. Whereas the $\mathrm{I}_{\mathrm{OFF}}$ value of $\mathrm{n}$-JLDGVM is increased by approximately $98 \%$ as the $\mathrm{N}_{\mathrm{sd}}$ is increased. This indicates that higher concentration of $\mathrm{N}_{\mathrm{sd}}\left(>10^{18} \mathrm{~cm}^{13}\right)$ is required to significantly change both ION and IOFF characteristic for p-JLDGVM. Figure 11 shows the impact of S/D doping upon the SS characteristic. It is observed that the SS values for n-JLDGVM are linearly increased as the $\mathrm{N}_{\text {sd }}$ is increased. However, the SS values for p-JLDGVM remains constant as the $\mathrm{N}_{\mathrm{sd}}$ is increased. Similar to $\mathrm{N}_{\mathrm{ch}}, \mathrm{N}_{\mathrm{sd}}$ do not contribute a significant impact on the SS characteristic for p-JLDGVM. The SS value of p-JLDGVM is more steep than the n-JLDGVM due to an extremely lower $I_{O F F}$ value in which the amount of $I_{D}$ increased per decade below the $\mathrm{V}_{\mathrm{TH}}$ in the p-JLDGVM is higher than the n-JLDGVM. As a result, the ON/OFF ratio of p-JLDGVM is observed to be much better than the n-JLDGVM.

It is observed that the SS values for n-JLDGVM are linearly increased as the $\mathrm{N}_{\mathrm{sd}}$ is increased. However, the SS values for p-JLDGVM remains constant as the $\mathrm{N}_{\mathrm{sd}}$ is increased. Similar to $\mathrm{N}_{\mathrm{ch}}, \mathrm{N}_{\mathrm{sd}}$ do not contribute a significant impact on the SS characteristic for p-JLDGVM. The SS value of p-JLDGVM is more steep than the n-JLDGVM due to an extremely lower $I_{O F F}$ value in which the amount of $I_{D}$ increased per decade below the $\mathrm{V}_{\mathrm{TH}}$ in the p-JLDGVM is higher than the n-JLDGVM. As a result, the ON/OFF ratio of p-JLDGVM is observed to be much better than the n-JLDGVM. 


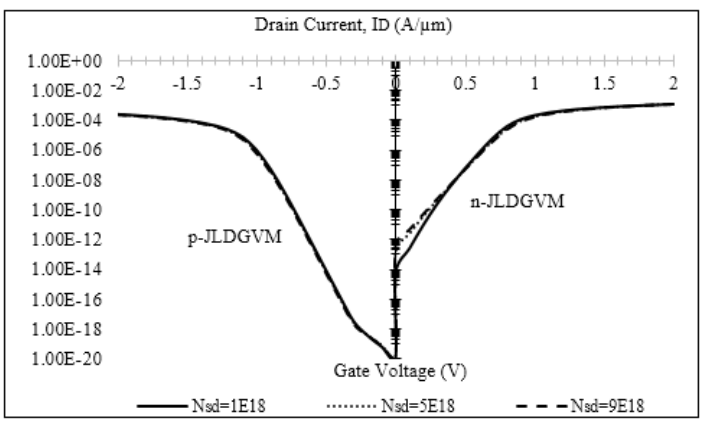

Figure 10. Graph of the subthreshold drain current $\left(\mathrm{I}_{\mathrm{D}}\right)$-gate voltage $\left(\mathrm{V}_{\mathrm{G}}\right)$ at $\mathrm{V}_{\mathrm{D}}=1.0 \mathrm{~V}$ with different concentration of source/drain doping

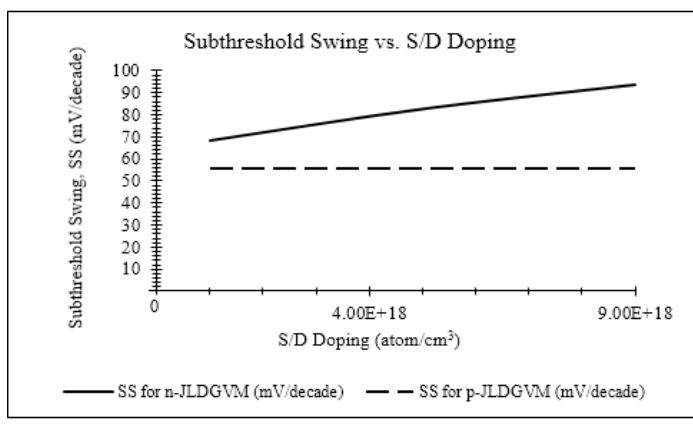

Figure 11. Subthreshold swing vs. s/d doping

\subsection{Metal-gate work function (WF)}

Apart from doping concentration, the work function (WF) also plays an important role to tune the $\mathrm{V}_{\mathrm{TH}}$ value in junctionless MOSFETs [13, 26-28]. The WF significantly depends on the distribution of atoms at the surface of channel material, where a specific material has a fixed WF value [29]. Previously, the application of different metal-gate work functions in JLDGVM has been reported [22], stating that the $\mathrm{I}_{\mathrm{D}}$ of n-JLDGVM was increased as the WF was decreased. This statement supports the current study about the variation effects of WF on the $\mathrm{I}_{\mathrm{D}}-\mathrm{V}_{\mathrm{G}}$ characteristic for both n-JLDGVM and p-JLDGVM devices, as depicted in Figure 12. It is observed that the $I_{D}$ for $n-J L D G V M$ is increased by $15 \%$ as the WF is reduced from $4.7 \mathrm{eV}$ to $4.5 \mathrm{eV}$. Such occurrence is mainly due to the early depletion of the channel surface between the two metal-gates that increases the $I_{D}$ without enhancing the channel concentration. However, reverse trend of $I_{D}$ variation has been observed in p-JLDGVM, where the value of $I_{D}$ is increased by $20 \%$ as the WF is increased from $4.5 \mathrm{ev}$ to $4.7 \mathrm{eV}$. Hence, it can be concluded that the WF value is inversely proportional to the $\mathrm{I}_{\mathrm{D}}$ variation in $\mathrm{n}-\mathrm{JLDGVM}$, while the WF value is directly proportional to $\mathrm{I}_{\mathrm{D}}$ variation in $\mathrm{p}-\mathrm{JLDGVM}$.

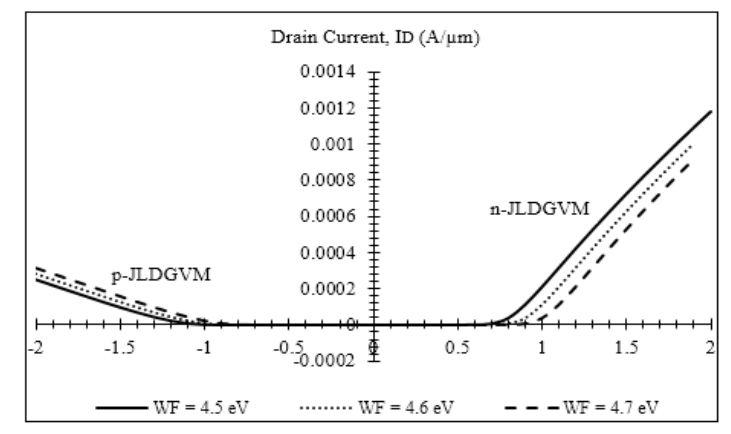

Figure 12. Graph of the drain current $\left(\mathrm{I}_{\mathrm{D}}\right)$-gate voltage $\left(\mathrm{V}_{\mathrm{G}}\right)$ at $\mathrm{V}_{\mathrm{D}}=1.0 \mathrm{~V}$ with different work functions

The combination of tungsten silicide and hafnium dioxide as a metal-gate and dielectric material respectively has improved the performance of JLDGVM in term of $\mathrm{I}_{\mathrm{D}}$, as well as $\mathrm{I}_{\mathrm{OFF}}$, as presented in Figure 13. It is observed that the IOFF value for n-JLDGVM is tremendously reduced by $99 \%$ as the WF is increased from $4.5 \mathrm{eV}$ to $4.7 \mathrm{eV}$. Similar to $\mathrm{I}_{\mathrm{D}}$ variation, the opposite trend is observed in the p-JLDGVM as IOFF value for p-JLDGVM is enormously reduced by approximately $97 \%$ as the WF is decreased from $4.7 \mathrm{eV}$ to $4.5 \mathrm{eV}$. From sub- $\mathrm{I}_{\mathrm{D}}-\mathrm{V}_{\mathrm{G}}$ characteristic, it can be concluded that the $\mathrm{WF}$ value is inversely proportional to the IOFF variation in n-JLDGVM, while the WF value is directly proportional to IOFF variation in p-JLDGVM. Thus, the performance of JLDGVM should be reasonably controlled with a proper tuning of the metal-gate WF. Figure 14 shows the impact of WF upon the SS variation in both n-JLDGVM and p-JLDGVM. It is observed that any changes in WF value do not contribute a significant impact on the SS value of both devices, since the SS values are almost constant as the WF is varied from 4.5 to $4.7 \mathrm{eV}$. This result further signifies that the elimination of poly depletion effect due to the introduction of high- $k /$ metal-gate stack in the JLDGVM architecture do provide better short channel effects (SCE) suppresion. 


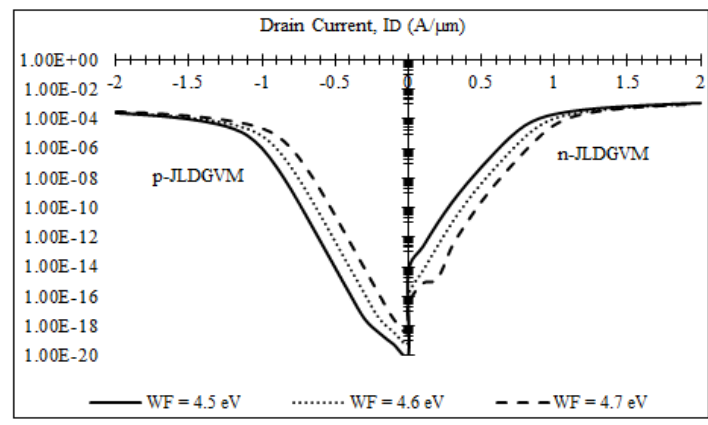

Figure 13. Graph of the the subthreshold drain current $\left(\mathrm{I}_{\mathrm{D}}\right)$-gate voltage $\left(\mathrm{V}_{\mathrm{G}}\right)$ at $\mathrm{V}_{\mathrm{D}}=1.0 \mathrm{~V}$ with different work functions

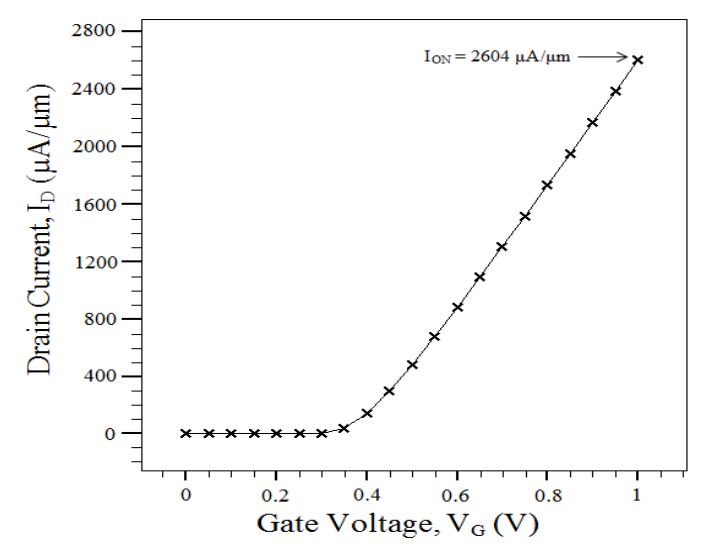

Figure 15. I-V curve at $\mathrm{V}_{\mathrm{D}}=0.5 \mathrm{~V}$ for $\mathrm{n}-\mathrm{JLDGVM}$ device with $\mathrm{WF}=4.5 \mathrm{eV}$

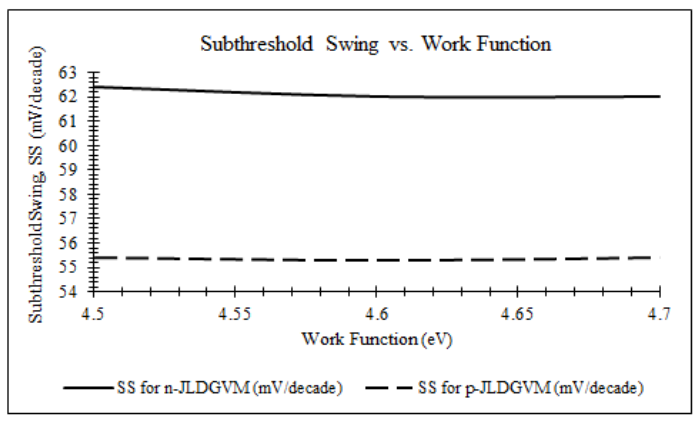

Figure 14. Subthreshold Swing vs. Work Function

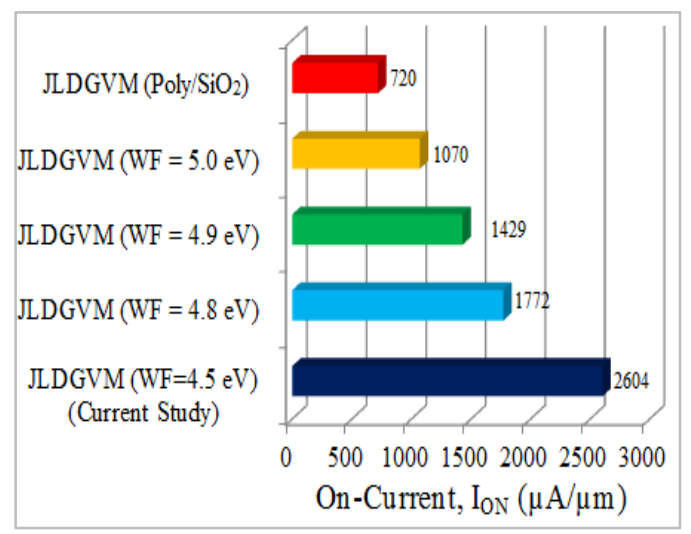

Figure 16. Benchmark of n-JLDGVM device with other junctionless vertical devices with multiple WF magnitude [18]

From the overall results, it can be concluded that the investigated geometrical and process parameter adjustments are very sensitive to the JLDGVM behavior. Although, the process parameter adjustment can boost the carrier mobility, its current improvement would be marginal when compared with geometrical parameter. For comparative purpose, the n-type device with $\mathrm{WF}=4.5 \mathrm{eV}$ was re-simulated at a constant $\mathrm{V}_{\mathrm{D}}=0.5 \mathrm{~V}$ as the $\mathrm{V}_{\mathrm{G}}$ is shifted from $0 \mathrm{~V}$ to $1 \mathrm{~V}$. The $\mathrm{I}_{\mathrm{ON}}$ magnitude of the $\mathrm{n}$-JLDGVM device with $\mathrm{WF}=4.5 \mathrm{eV}$ can be measured at $\mathrm{V}_{\mathrm{G}}=1 \mathrm{~V}$ as depicted in Figure 15. Figure 16 summarizes the comparison of the $\mathrm{I}_{\mathrm{ON}}$ magnitude of this current study with vertical double-gate transistors with multiple WF magnitude from previous studies [22]. Based on the comparison, it is observed that the n-JLDGVM device (current study) has demonstrated the highest ION magnitude compared to other devices. The trend of ION magnitude is in agreement with the previous studies as the lower WF do contribute a higher I ON magnitude. Tuning the metal-gate WF in the range of 4.5-4.7 eV seems to be an effective method in attaining much better ON and OFF state current as well as ON/OFF ratio. In addition, doping concentration can be also adjusted to tune JLDGVM performance, yet the trend of its impact upon $\mathrm{I}_{\mathrm{D}}-\mathrm{V}_{\mathrm{G}}$ characteristic has to be well considered. Thus, controlling the geometrical and process design is very crucial in order to obtain better device performance. For future work, several optimization approaches [30-33] could be employed to control and optimize the geometrical and process variation for improved JLDGVM performance.

\section{CONCLUSION}

Multiple electrical characteristics of the JLDGVMs are highly dependent on the JLDGVM design considerations. Considering the scaling of JLDGVM, it is concluded that $\mathrm{L}_{\mathrm{ch}}$ scaling plays a crucial role in tuning the electrical characteristics such as $\mathrm{I}_{\mathrm{ON}}$, $\mathrm{I}_{\mathrm{OFF}}$ and $\mathrm{V}_{\mathrm{TH}}$. Pillar design is also important and most preferable if the silicon pillar has a vertically ultra-thin shape so that the gate controllability can be fully 
maximized. Despite of having high performance due to the fully-depleted channel, JLDGVM also suffers undesired degradation effect such as SCEs. Therefore, with process design consideration, these effects can be well monitored and excellent gate controllability could be gained, thus enabling JLDGVM design to be further optimized for better performance, especially from driveability and leakage perspective. Application of doping tuning approach and WF engineering are crucial for attaining desired threshold and lower leakage design. For future work, the JLDGVM design can be further optimized via appropriate optimization methods in order to improve the electrical characteristics. Thus, it is suggested that all of the parameters dependencies on JLDGVM performance should be carefully considered since the junctionless configuration is significantly associated with the geometrical and process parameters.

\section{ACKNOWLEDGEMENTS}

The authors would like to thank to the Ministry of Higher Education (MOHE) of Malaysia, Mybrain15 and Centre for Telecommunication Research and Innovation (CeTRI), Faculty of Electronics and Computer Engineering (FKEKK), Universiti Teknikal Malaysia Melaka (UTeM) for sponsoring this research study under research grant (FRGS/1/2017/TK04/FKEKK-CeTRI/F00335).

\section{REFERENCES}

[1] B. Subrahmanyam and M. J. Kumar, "Recessed source concept in nanoscale vertical surrounding gate (VSG) MOSFETs for controlling short-channel effects," Physica E: Low-Dimensional Systems and Nanostructures, vol. 41, pp. 671-676, 2009.

[2] T. Uchino, et al., "Improved vertical MOSFET performance using an epitaxial channel and a stacked siliconinsulator structure," Semiconductor Science and Technology, vol/issue: 27(6), pp. 062002, 2012.

[3] M. Wu, et al., "The Optimal Design of Junctionless Transistors with Double-Gate Structure for reducing the Effect of Band-to-Band Tunneling," JSTS: Journal of Semiconductor Technology and Science, vol/issue: 13(3), pp. 245-251, 2013.

[4] M. Khaouani and A. G. Bouazza, "Impact of multiple channels on the Characteristics of Rectangular GAA MOSFET," International Journal of Electrical and Computer Engineering, vol/issue: 7(4), pp. 1899-1905, 2017.

[5] N. Shehata, et al., "3D Multi-Gate Transistors: Concept, Operation, and Fabrication," Jounal of Electrical Engineering, vol. 3, pp. 1-14, 2015.

[6] M. M. A. Hakim, et al., "Self-Aligned Silicidation of Surround Gate Vertical MOSFETs for Low Cost RF Applications," IEEE Transactions on Electron Devices, vol/issue: 57(12), pp. 3318-3326, 2010.

[7] M. Masahara, et al., "Ultrathin Channel Vertical DG MOSFET Fabricated by Using Ion-Bombardment-Retarded Etching," IEEE Transactions on Electron Devices, vol/issue: 51(12), pp. 2078-2085, 2004.

[8] N. F. Kosmani, et al., "A comparison of performance between double-gate and gate-all-around nanowire mosfet," Indonesian Journal of Electrical Engineering and Computer Science, vol/issue: 13(2), pp. 801-807, 2019.

[9] H. Jung, "Threshold voltage roll-off for sub-10 nm asymmetric double gate," International Journal of Electrical and Computer Engineering, vol/issue: 9(1), pp. 163-169, 2019.

[10] A. F. Roslan, et al., "30nm DG-FinFET 3D Construction Impact towards Short Channel Effects," Indonesian Journal of Electrical Engineering and Computer Science, vol/issue: 12(3), pp. 1358-1365, 2018.

[11] T. Solankia and N. Parmar, "A Review paper: A Comprehensive study of Junctionless transistor," in National Conference on Recent Trends in Engineering \& Technology, pp. 1-5, 2011.

[12] Vikkee and A. Nandi, "Improved Analytical Modeling for Junctionless," International Journal of Electrical, Electronics and Data Communication, vol/issue: 3(7), pp. 19-21, 2015.

[13] A. K. Gupta, et al., "To Study and Characterisation of N N + N Nanowire Transistor (Junctionless) using 2D ATLAS Simulator," International Journal of Current Engineering and Technology, vol/issue: 4(3), pp. 2203-2206, 2014.

[14] A. K. Mandia and A. K. Rana, "Performance Enhancement of Double Gate Junctionless Transistor Using High-K Spacer and Models," Proceedings of 11th IRF International Conference, vol. 8, pp. 8-11, 2014.

[15] S. R. Mulmane, et al., "Simulation of Nanoscale Fully Depleted EJ- SOI Junctionless MOSFET for High Performance," International Journal of Industrial Electronics and Electrical Engineering, vol/issue: 4(6), pp. 34-37, 2016.

[16] R. Norani, "A Dynamic Simulation on Single Gate Junctionless Field Effect Transistor Based on Genetic Algorithm," Advances in Computer Science: An International Journal, vol/issue: 3(5), pp. 140-145, 2014.

[17] M. Puttaveerappa, et al., "Junctionless FETs with a Fin Body for Multi-V TH and Dynamic Threshold Operation," IEEE Transactions on Electron Devices, vol/issue: 65(8), pp. 3535-3542, 2018.

[18] K. Biswas, et al., "Fin shape influence on analog and RF performance of junctionless accumulation-mode bulk FinFETs," Microsystem Technologies, vol/issue: 24(5), pp. 2317-2324, 2018.

[19] S. C. Wagaj, et al., "Performance analysis of shielded channel double-gate junctionless and junction MOS transistor," International Journal of Electronics Letters, vol/issue: 6(2), pp. 192-203, 2018.

[20] F. Larki, et al., "Electronic Transport Properties of Junctionless Lateral Gate Silicon Nanowire Transistor Fabricated by Atomic Force Microscope Nanolithography," Microelectronics and Solid State Electronics 2012, vol/issue: 1(1), pp. 15-20, 2012.

Geometric and process design of ultra-thin junctionless double gate vertical MOSFETs (K. E. Kaharudin) 
[21] J. P. Colinge, et al., "A Simulation Comparison between Junctionless and Inversion-Mode MuGFETs," ECS Transactions, vol/issue: 35(5), pp. 63-73, 2011.

[22] J. Rahul, et al., "Effects of Metal Gate Electrode and HfO 2 in Junctionless Vertical Double Gate MOSFET," International Journal of Scientific Engineering and Technology, vol/issue: 3(5), pp. 671-674, 2014.

[23] Silvaco, "Silvaco ATLAS manual Device Simulation Software," 2006.

[24] V. K. Yadav and A. K. Rana, "Impact of Channel Doping on DG-MOSFET Parameters in Nano Regime-TCAD Simulation," International Journal of Computer Applications, vol/issue: 37(11), pp. 36-41, 2012.

[25] M. Rahman, et al., "Metal-gated junctionless nanowire transistors," International Conference on Simulation of Semiconductor Processes and Devices, pp. 8-9, 2012.

[26] B. Lakshmi and R. Srinivasan, "Effect of Process Parameter Variation on $\mathrm{ft}$ in Coventional and Junctionless GateAll-Around Devices," Journal of Engineering Science and Technology, vol/issue: 10(8), pp. 994-1008, 2015.

[27] K. P. Londhe and Y. V. Chavan, "A Novel Double Gate Junction-less MOSFET Using Germanium," International Conference on Innovative Trends in Engineering Research, pp. 115-118, 2016.

[28] J. Rahul, et al., "Performance evaluation of junctionless vertical double gate MOSFET," 2012 International Conference on Devices, Circuits and Systems, ICDCS 2012, pp. 440-442, 2012.

[29] F. A. Rezali, et al., "Performance and device design based on geometry and process considerations for $14 / 16-\mathrm{nm}$ FinFETs stress engineering," IEEE Transactions on Electron Devices, vol/issue: 63(3), pp. 974-981, 2016.

[30] K. E. Kaharudin, et al., "Application of Taguchi-based Grey Fuzzy Logic for Simultaneous Optimization in TiO2/WSix-based Vertical double-gate MOSFET," Journal of Telecommunication, Electronic and Computer Engineering, vol/issue: 9(2-13), pp. 23-28, 2017.

[31] K. E. Kaharudin, et al., "Impact of Different Dose, Energy and Tilt Angle in Source / Drain Implantation for Vertical Double Gate PMOS Device," Journal of Telecommunication, Electronic and Computer Engineering, vol/issue: 8(5), pp. 23-28, 2016.

[32] K. E. Kaharudin, et al., "Implementation of Taguchi Method for Lower Drain Induced Barrier Lowering in Vertical Double Gate NMOS Device," Journal of Telecommunication, Electronic and Computer Engineering, vol/issue: 8(4), pp. 11-16, 2016.

[33] K. E. Kaharudin, et al., "Comparison of Taguchi Method and Central Composite Design for Optimizing Process Parameters in Vertical Double Gate MOSFET," ARPN Journal of Engineering and Applied Sciences, vol/issue: 12(19), pp. 5578-5590, 2017.

\section{BIOGRAPHIES OF AUTHORS}

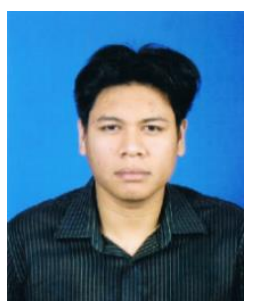

K. E. Kaharudin received $\mathrm{PhD}$ in Electronic Engineering and $\mathrm{M}$. Eng degree in Computer Engineering from Technical University of Malaysia Melaka (UTeM), in 2017 and 2013 respectively. His $\mathrm{PhD}$ project focused on the process optimization of vertical double gate MOSFET. His research's interests include CMOS design, microelectronics, semiconductors, engineering optimization and artificial intelligence. Recently, his efforts emphasize on the simulation design of Junctionless MOSFET, silicon-on-insulator (SOI) MOSFET, high-k/metalgate stack technology and design of experiment (DoE).

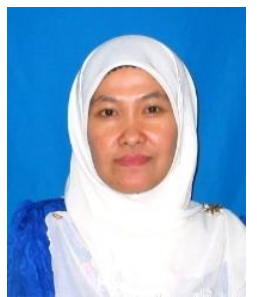

F. Salehuddin received the B.Sc. degree in electrical engineering (Communication) from Universiti Teknologi Mara (UiTM), Malaysia in 2001 and the M.Sc. degree in Electrical, Electronic and System Engineering from Universiti Kebangsaan Malaysia, in 2003. She received the Ph.D. degree in Microelectronics Engineering from Universiti Tenaga Nasional (UNITEN), Malaysia in 2012. She joined Universiti Teknikal Malaysia Melaka (UTeM) in December 2001 as a tutor and is currently a senior lecturer at Faculty of Electronic and Computer Engineering (FKEKK), UTeM. Her research interest includes process and device simulation of nanoscale MOSFETs device, advanced CMOS design, optimization approach (DOE) and process parameter variability.

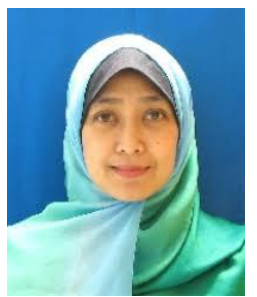

A. S. M. Zain received the B. Eng degree in electrical, Electronic and System engineering and M. Sc in Microelectronics from National University of Malaysia (UKM), Malaysia She received the Ph.D. degree in Nanoelectronics Engineering from University of Glasgow, UK in 2013. She is currently working as a senior lecturer at Faculty of Electronic and Computer Engineering (FKEKK), Universiti Teknikal Malaysia Melaka (UTeM). Her research interest includes Nanoscale Device Design and Simulation, Variability and Reliability of Emerging Technology Devices, IC Design for Biomedical Applications. 


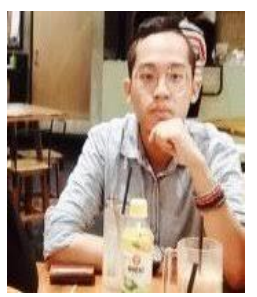

Ameer F. Roslan received by. Sc. Degree in Telecommunication Engineering from Technical University of Malaysia Melaka (UTeM), in 2017. He is currently pursuing his MSc degree in electronics and doing research on the DG-FinFET device at Centre for Telecommunication Research and Innovation, Faculty of Electronics and Computer Engineering, Technical University of Malaysia Melaka (UTeM). His research interest includes the DG-FinFET architecture and statistical 